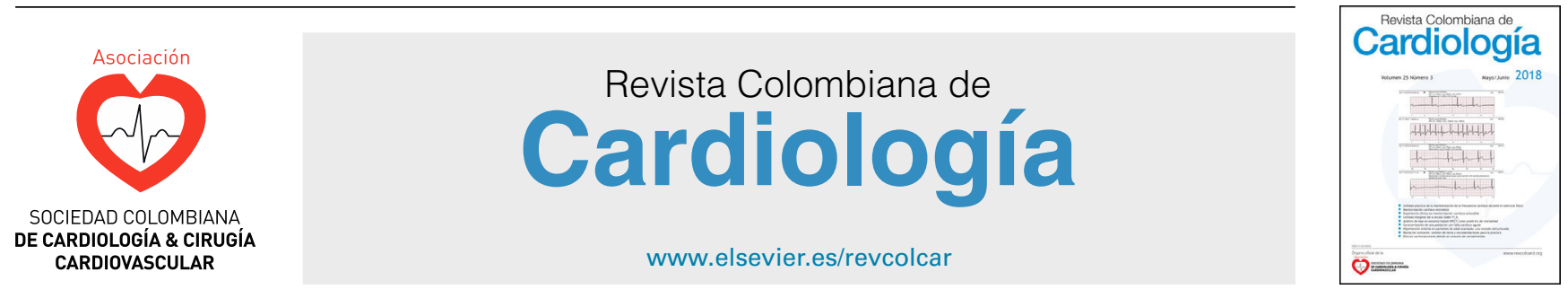

CARDIOLOGÍA DEL ADULTO - REVISIÓN DE TEMAS

\title{
La repolarización auricular y sus implicaciones clínicas
}

\section{Mauricio Duque $^{a, *}$, David Aristizábal-Colorado ${ }^{\mathrm{b}}$, Danilo Weir ${ }^{\mathrm{b}}$, María C. Gaviriaa y William Uribe ${ }^{a}$}

a Universidad CES, CES Cardiología, Medellín, Colombia

b Fundación Universitaria San Martín, Medellín, Colombia

Recibido el 10 de enero de 2018; aceptado el 29 de mayo de 2019

Disponible en Internet el 4 de noviembre de 2019

\author{
PALABRAS CLAVE \\ Aurícula; \\ Electrocardiograma
}

\section{KEYWORDS}

Atrium;

Electrocardiogram

\begin{abstract}
Resumen La onda P en el electrocardiograma (ECG) representa exclusivamente la despolarización de las aurículas, puesto que, la repolarización auricular queda oculta en el QRS. Sin embargo, cuando la repolarizacón auricular se hace evidente en el ECG se convierte en un elemento clave para el diagnóstico de algunas enfermedades. Diferente a lo que sucede en la repolarización ventricular, la repolarización auricular sigue el mismo sentido de la despolarización, pues sigue un sentido cráneo-caudal y se da desde el epicardio hacia el endocardio.

( ) 2019 Sociedad Colombiana de Cardiología y Cirugía Cardiovascular. Publicado por Elsevier España, S.L.U. Este es un artículo Open Access bajo la licencia CC BY-NC-ND (http:// creativecommons.org/licenses/by-nc-nd/4.0/).
\end{abstract}

\section{Atrial repolarisation and its clinical implications}

Abstract The electrocardiogram (ECG) wave represents exclusively the depolarization of the atria, so auricular repolarization is hidden in the QRS. However, when auricular repolarization is evident in the ECG, it becomes a key element in the diagnosis of some diseases. Different from what happens in ventricular repolarization, auricular repolarization follows the same sense of depolarization, since it follows a cranial-caudal direction and is from the epicardium to the endocardium.

( 2019 Sociedad Colombiana de Cardiología y Cirugía Cardiovascular. Published by Elsevier España, S.L.U. This is an open access article under the CC BY-NC-ND license (http:// creativecommons.org/licenses/by-nc-nd/4.0/).

\footnotetext{
* Autor para correspondencia.

Correo electrónico: mauricioduquemd@gmail.com (M. Duque).
}

\section{Introducción}

La onda $P$ en el electrocardiograma (ECG) representa específicamente la despolarización auricular y sugiere la despolarización del nodo sinusal. En términos generales, la 
despolarización avanza desde el nodo sinusal, ubicado en la región superior y posterior de la aurícula derecha, y se propaga hacia la porción inferior y lateral para lograr así la despolarización completa de la aurícula derecha, continuar con la aurícula izquierda y llegar hasta el nodo aurículo ventricular (AV). En esta propagación se usan los tractos internodales y el haz de Bachmann ${ }^{1-3}$.

Parte de esta actividad se observa en el ECG: la despolarización. En la mayoría de las ocasiones la repolarización auricular no se evidencia en el ECG debido a que es enmascarada por el gran potencial correspondiente al QRS ${ }^{1-5}$.

Al interpretar las ondas en un ECG siempre se deben evaluar la onda $\mathrm{P}$ y el segmento $\mathrm{PR}$ que representa el retraso fisiológico de la conducción $\mathrm{AV}$, puesto que es fundamental para identificar diferentes patologías, como trastornos del ritmo o dilatación de las cavidades, y en este sentido durante la interpretación de la onda $P$ se debe tener en cuenta el concepto de repolarización, el cual, al parecer, no es tan inocuo y es realmente importante en varias enfermedades ${ }^{4,6}$. En esta revsión se busca hacer un acercamiento a los conceptos de repolarización auricular y los hallazgos patológicos que aportan al diagnóstico de algunas enfermedades en la práctica clínica.

\section{Métodos}

Se realizó una búsqueda no sistemática de la literatura en PubMed, con los términos MeSh "Repolarization AND Atrium', Se filtraron los resultados por "FullText", lo cual dio como resultado inicial 522 ítems. Bajo estos mismos criterios se realizó la búsqueda en EMBASE, la cual arrojó 349 resultados, además de libros de texto de referencia en el área. La selección de los artículos finales se hizo a criterio de los autores. Se excluyeron todos los resultados duplicados, cartas al editor y artículos en idiomas diferentes al inglés o al español.

\section{Resultados}

Se encontraron 871 ítems con fechas de publicación entre 1956 y 2016, los cuales se evaluaron cualitativamente por medio de relevancia en título y resumen, para su posterior revisión a fondo. Luego de evaluar estos resultados y al aplicar los criterios de inclusión y exclusión, finalmente fueron elegidos 38 artículos, los cuales se revisaron exhaustivamente para la redacción de este artículo; en ese sentido, se exploraron referencias importantes de los mismos y libros de textos relevantes en el tema.

\section{Despolarización y repolarización auricular}

En primer lugar, se debe entender que la despolarización y la repolarización obedecen a diferentes fenómenos de intercambio iónico en las células cardíacas y esto se traduce en el potencial de acción del cardiomiocito, que por las propiedades histológicas del tejido cardíaco permite que la corriente se propague de manera longitudinal y transversal ${ }^{5,7}$. Este potencial de acción, o al menos la despolarización auricular, se observa mediante el ECG de superficie a través de la onda $\mathrm{P}^{1-4}$. Cabe resaltar que la repolarización auricular, debido a su voltaje $(100 \mu \mathrm{V})$ y tiempo de inicio, queda enmascarada en el ECG por la despolarización ventricular, la cual genera mayor voltaje y da como resultado el complejo QRS ${ }^{1-4,6}$. Posterior a esto se produce la repolarización ventricular: se genera un vector de repolarización en dirección contraria a la despolarización, dado que pese a que la despolarización inicia en el endocardio, la repolarización también debería empezar allí, pero hay diferencias entre la duración de los potenciales de acción entre el endocardio y el epicardio, pues es más largo en el endocardio y más corto en el epicardio; además, la irrigación de los ventrículos se da principalmente en diástole y en el endocardio hay un aumento en la presión diastólica final, lo cual hace al endocardio más vulnerable y se comportaría en cierto caso como isquémica, sin estarlo. Por consiguiente, la repolarización ventricular inicia desde el epicardio, por su mayor flujo sanguíneo y su potencial de acción más corto, hecho que genera un vector con la misma dirección del vector del $\mathrm{QRS}$, lo que se conoce como la onda $\mathrm{T}_{\text {del }} \mathrm{ECG}^{1-3}$.

De otro lado, contrario a los ventrículos, en las aurículas el vector de repolarización va en el mismo sentido al de la despolarización, de endocardio a epicardio, pues no hay sobrecarga de presión en las aurículas. No obstante, conviene recordar que la repolarización es una corriente negativa neta, diferente a la despolarización, la cual da cargas positivas (ingreso de sodio al cardiomiocito), fenómeno que se explica por el menor grosor de la pared auricular en comparación con la pared ventricular y debido a que la oxigenación del endocardio y del subendocardio auricular se da por difusión, por lo cual se genera una deflexión negativa en el $\mathrm{ECG}^{1-4}$. En la figura 1 se exponen y comparan dichas ondas.

\section{Electrocardiografía de la repolarización auricular}

Pese a que la repolarización auricular (Ta) es un fenómeno que no se evidencia frecuentemente en el ECG, en algunos escenarios cuando se prolonga el intérvalo PR o existe disociación AV es posible observarla, sin ser un hallazgo patológico ${ }^{4,5,8-10}$. Cabe agregar que la Ta no solo se aprecia en estos casos, pues se encuentra descrita como "un platillo" que se observa en algunas ocasiones en presencia de un PR normal (120-200 ms), lo cual sustenta que el PR no siempre es la línea isoeléctrica en el ECG ${ }^{4,11,12}$ (figs. 2 y 3).

Es más común enfrentarse a la Ta en escenarios normales conforme aumenta el voltaje de estímulo del nodo sinusal y cuando cambia la frecuencia de estímulo, explicándose así, que en las pruebas de esfuerzo no sea extraño encontrar una depresión en el PR, que además puede prolongarse hasta el segmento ST en las derivadas inferiores durante esta prueba ${ }^{4,10,12}$, casos en los cuales fácilmente se puede malinterpretar dicho trazo y diagnosticar cambios isquémicos sin que necesariamente estén presentes ${ }^{4,13}$. De lo anterior se concluye que a mayor frecuencia cardiaca, mayor voltaje de la $\mathrm{P}$, y mayor pendiente en el PR, que a su vez incrementa el riesgo de alterar el $\mathrm{ST}^{4,6,14}$. Con esto presente, se resalta que algunas $P$ de origen ectópico generan mayor voltaje en comparación con una $P$ de origen sinusal, lo cual puede favorecer alteraciones en el $\mathrm{ST}^{4,6,9,15}$. Si se considera lo previo con las altas frecuencias cardíacas de las arritmias 


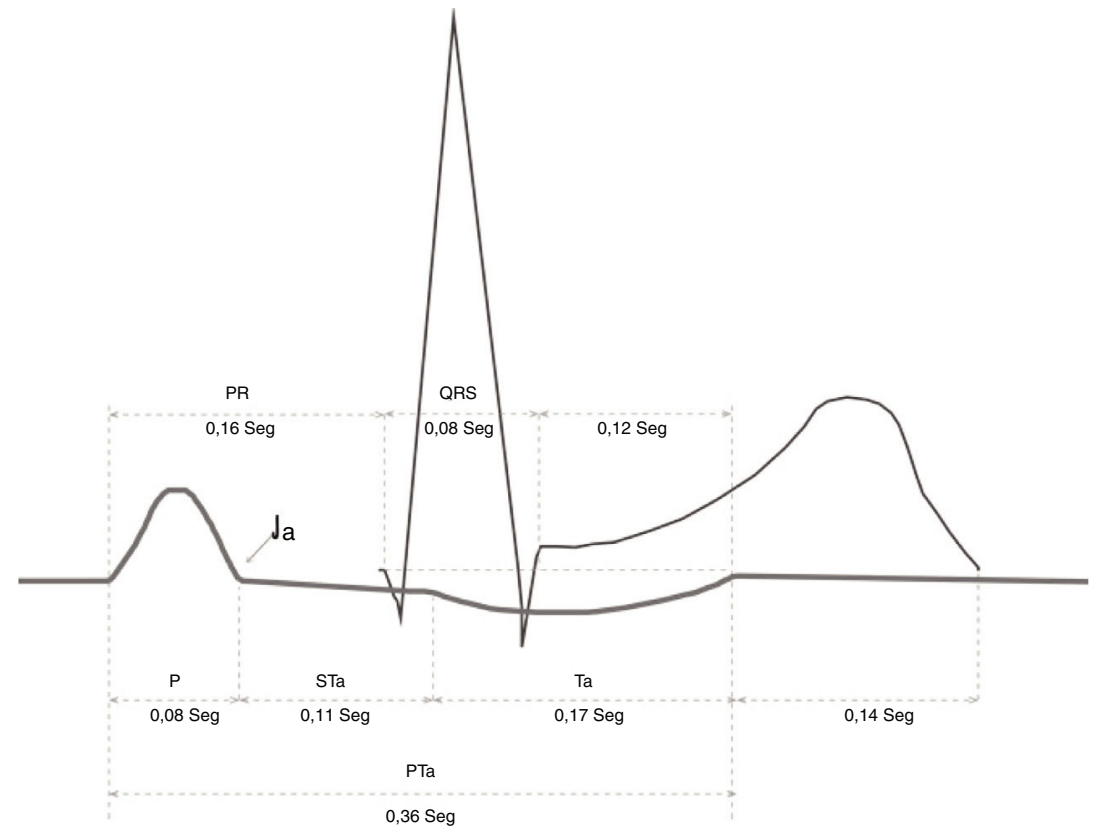

Figura 1 Potenciales de acción en el ECG. STa: segmento entre el final de la P hasta la onda de repolarización auricular. Ta: onda de repolarización auricular. PTa: sumatoria de STa y Ta. Modificado de Alderete, 1999.

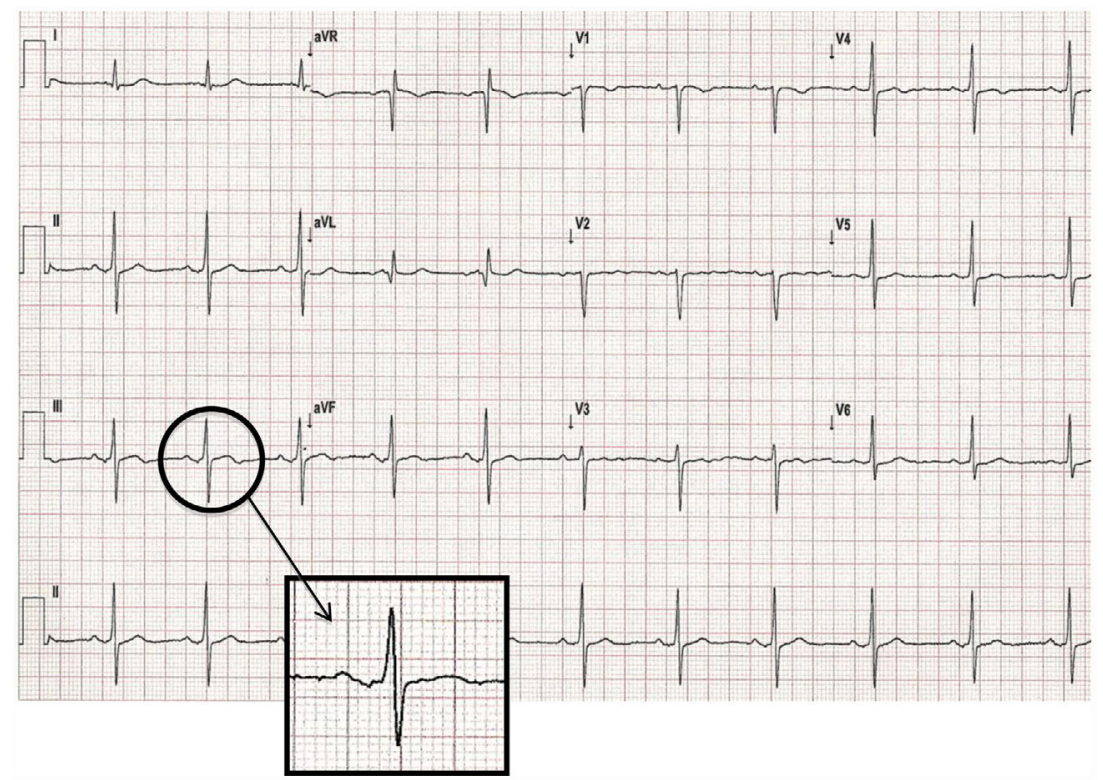

Figura 2 Electrocardiograma que demuestra alteraciones en la repolarización representadas por muesca en la porción descendente de la onda P, visibles en las derivadas DI, DII, DIII y aVL.

supraventriculares, se puede explicar, en cierta medida, la alteración del ST en los trazos de dichos eventos ${ }^{4,9,14,15}$.

\section{Valoración de la onda de repolarización auricular $(\mathrm{Ta})$ y sus segmentos}

El PTa está definido como la suma del tiempo que transcurre desde el final de la onda $\mathrm{P}$ hasta el final de la onda Ta. Para valorar el Ta y PTa se ha demostrado que las derivadas convencionales son subóptimas. Ihara et al., realizaron un estudio en el que se hizo un mapeo de 64 derivaciones en el tórax de 75 voluntarios sanos y lograron determinar que el lugar idóneo para la evaluación del proceso eléctrico auricular se encuentra en V2 y el área pectoral superior derecha que corresponde al lugar donde se ubica el electrodo del desfibrilador ${ }^{6}$.

Un aspecto fundamental de la repolarización auricular es su relación directa, al igual que en los ventrículos, con la presencia de arritmias del sincitio cardiaco. Dicho lo 


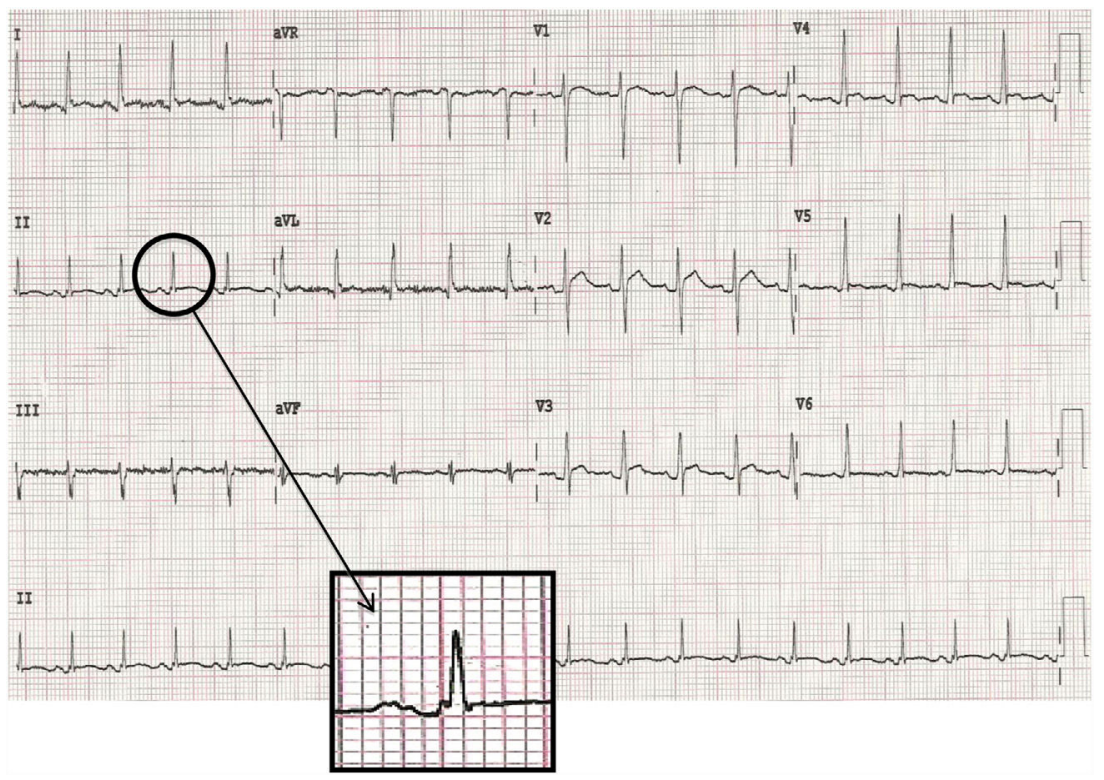

Figura 3 Electrocardiograma en el que se aprecian alteraciones en la repolarización representadas por onda P bifásica con muesca en la porción ascendente que se observan con mayor claridad al amplificar un fragmento de DII.

anterior, se evidencia que las alteraciones en la repolarización cardiaca son de gran trascendencia y por esto sus tiempos deberían ser cuantificados. Al respecto, Debbas et al. proponen el uso de una fórmula para la corrección del PTa: ${ }^{5}$

PTa Corregido $=$ PTa $-0,24$ (PP-1000)

En consecuencia, se ha definido que el PTa se expresa en términos de milisegundos; el PTa presenta casi todas las mismas propiedades en el sincitio auricular que el QT en el sincitio ventricular, excepto una característica puntual: las aurículas son principalmente mediadas por el tono parasimpático, mientras que los ventrículos por el simpático, lo cual marca diferencias entre ambos intervalos en situaciones como el ejercicio ${ }^{5,13}$.

\section{Relevancia clínica de la repolarización auricular}

La importancia del PTa y su duración se traduce directamente en la duración normal del potencial de acción auricular, el cual tiene efecto protector para las reentradas anatómicas, evitando así latidos ectópicos, FA paroxística, entre otros ${ }^{11,16}$; sin embargo, este mecanismo no protege sobre las reentradas funcionales. De cualquier manera, la presencia de una repolarización auricular normal se traduce en mejores resultados a la hora de hacer ablaciones en diferentes enfermedades asociadas $^{17}$.

Una de las situaciones que cursa con alteración directa del Ta es el infarto auricular, entidad que tiene alta mortalidad por su asociación con infarto masivo. Es importante destacar que dichos infartos raramente se presentan en la aurícula izquierda, dado que existe un mayor baño endocárdico de sangre altamente oxigenad que llega directamente de la circulación pulmonar y se enfrenta a un menor grosor de la pared auricular, lo que evita dicha condición ${ }^{4,15}$. El infarto auricular cursa con un Ta que tiende a la depresión y se observa en el ECG como un PR descendente, similar a las alteraciones de la pericarditis; en ambos casos esto se explica por la alteración directa en la repolarización auricular ${ }^{4,13}$. También se han descrito casos en los que se atribuye a una "pseudo" prolongación del ST a prolongaciones directas del Ta, condición que quizás podría contribuir al diagnóstico de canalopatías.

\section{Conclusión}

La repolarización auricular es un fenómeno que pocas veces es evidente en el ECG de superficie, por consiguiente, cuando se visualiza en el ECG es de gran relevancia clínica, puesto que se comporta como una clave diagnóstica en algunas enfermedades.

\section{Financiación}

Ninguna.

\section{Conflictos de interés}

Ninguno.

\section{Bibliografía}

1. Goldberger AL, Goldberger ZD, Alexei S. Goldberger's Clinical Electrocardiography: A Simplified Aproach. 8 th. ed. Philadelphia: Elsevier; 2013.

2. Uribe W. Electrocardiografía clínica. De lo básico a lo complejo. 1a . ed Bogotá: Distribuna LTDA; 2014.

3. Bayés de Luna A. Clinical electrocardiography. 4 th. ed. Oxford, UK: Wiley-Blackwell; 2010. 
4. Childers R. Atrial repolarization: its impact on electrocardiography. J Electrocardiol. 2011;44:635-40.

5. Debbas NMG, Jackson SHD, de Jonghe D, Robert A, Camm AJ. Human atrial repolarization: effects of sinus rate, pacing and drugs on the surface electrocardiogram. J Am Coll Cardiol. 1999;33:358-65.

6. Ihara Z, van Oosterom A, Hoekema R. Atrial repolarization as observable during the $\mathrm{PQ}$ interval. J Electrocardiol. 2006;39:290-7.

7. Firek L, Giles WR. Outward currents underlying repolarization in human atrial myocytes. Cardiovascular Research. 1995;30:31-8.

8. Abramson DI, Fenichel NM, Shookhoff C. A study of electrical activity in the auricles. Am Heart J. 1938;15:471-81.

9. Sodi-Pallares D, Soberón J, Cabrera E, De la Cruz M. Consideraciones en relación a la repolarización auricular. Archivos Brasileiros de Cardiologia. 1948;1:113-27. Disponible en: http://www.cardiol.br/tunel/ago/new/008.pdf.

10. Holmqvist F, Carlson J, Platonov PG. Detailed ECG analysis of atrial repolarization in humans. Annals of Noninvasive Electrocardiology. 2009;14:13-8.

11. Hertervig E, Li Z, Kongstad O, Holm M, Olsson SB, Yuan S. Global dispersion of right atrial repolarization in healthy pigs and patients. Scandinavian Cardiovascular Journal. 2003;37:329-33.

12. Hayashi H, Okajima M, Yamada K. Atrial T(Ta) wave and atrial gradient in patients with $\mathrm{A}-\mathrm{V}$ block. Am Heart J. 1976;91:689-98.

13. Tranchesi J, Adelardi V, De Oliveira JM. Atrial Repolarization-Its Importance in Clinical Electrocardiography. Circulation. 1960;22:635.

14. Blankstein R, Childers R. Atrial repolarization causing psuedoprolongation of the QT Interval. Annals of Noninvasive Electrocardiology. 2008;13:317-8.

15. Langley P, Caldwell J. The atrial T wave: The elusive electrocardiographic wave exposed by a case of shifting atrial pacemaker. J Electrocardiol.49(4):557-9.

16. Verrier RL, Fuller $H$, Justo $F$, Nearing $B D$, Rajamani $S$, Belardinelli L. Unmasking atrial repolarization to assess alternans, spatiotemporal heterogeneity, and susceptibility to atrial fibrillation. Heart Rhythm. 2016;13: 953-61.

17. Ridler M-E, Lee M, McQueen D, Peskin C, Vigmond E. Arrhythmogenic consequences of action potential duration gradients in the atria. Canadian Journal of Cardiology. 2011;27:112-9. 